\title{
Reduction of population variability in protein expression: A control engineering approach.
}

\author{
Yadira Boada, Alejandro Vignoni, Jesús Picó \\ \{yaboa, alvig2, jpico\}@upv.es \\ Institut d'Automàtica i Informàtica Industrial, Universitat Politècnica de València, \\ Camino de Vera s/n, 46022 Valencia, España
}

\section{Resumen}

Controlling protein expression level is of interest in many applications. Yet, the stochastic nature of gene expression plays an important role and cannot be disregarded. We propose a gene synthetic circuit designed to control the mean gene expression in a population of cells and its variance. The circuit combines an intracellular negative feedback loop and quorum sensing based cell-to-cell communication system. Our in silico analysis using stochastic simulations reveals significant noise attenuation in gene expression through the interplay between quorum sensing and negative feedback, and explain their different roles for different noise sources. Preliminary in vivo results agree well with the computational results.

Palabras clave: cellular noise, noise attenuation, protein expression control, quorum sensing, feedback control.

\section{Introduction}

Noise is pervasive in the cellular mechanisms underlying gene expression [26]. It propagates to downstream genes at the single cell level, and eventually causes variation within an isogenic population $[25,19]$ that may determine the fate of individual cells and that of a whole population $[8,19]$.

At the gene level, noise can be traced back to intrinsic sources due to stochastic fluctuations in transcription and translation mechanisms, and extrinsic ones corresponding to gene independent fluctuations in protein expression due to external factors $[8,3,14]$. To minimize the deleterious effects of noise, cells have evolved different strategies at the single-cell level: from different transcription and translation efficiency so as to reduce translation burst rates in key genes [16] to more elaborated strategies, such as negative feedback regulation to reduce noise by shifting the noise spectrum to a higher frequency region [26]. Yet, cells live in communities, forming a population. At this level, extracellular signaling propagates intracellular stochastic fluctuations across the population [34]. Thus, cells have adapted their communication mechanisms in order to improve the signal-tonoise ratio [40]. One of such communication mechanisms is quorum sensing.

Quorum sensing (QS), initially discovered in $V$. fisheri and P. putida, is a cell-to-cell communication mechanism whereby bacteria exchange chemical signaling molecules, called autoinducers, whose external concentration depends on the cell population density. It is known that synchronization and consensus protect from noise [33]. Cells detect a threshold concentration of QS autoinducers and alter gene expression accordingly [10], driving the population as a whole to achieve a desired consensus gene expression level despite the individual noise of each member of the population. Cells consensus induced by QS is thought to reduce extrinsic noise by reducing the transmission of fluctuating signals in the low-frequency domain [35], enhances intrinsic stochastic fluctuations [34], and allows entrainment of a noisy population when faced to environmental changing signals [22]. Therefore QS seems an effective tool to control the phenotypic variability in a population of cells [40].

Phenotypic variability has important practical relevance in many applications in the areas of biomedicine, biotechnology and other branches of biological science [11] as the presence of heterogeneous subpopulations may have significant impact on the yield and productivity of industrial cultures $[21,9,2]$. Thus, improving homogeneity of protein expression in industrial cultures is a goal of economic relevance for microbial cell factory processes. that has traditionally been attempted either by optimizing environmental conditions in the culture or by careful selection of the strain. Open loop strategies based on sensitivity analysis have been used to provide guides as to how properly tune transcriptional and translational parameters so that the noise levels can be controlled while the mean values can be simultaneously adjusted to desired values [18]. While sensitivity analysis gives very valuable insights, open loop control is 


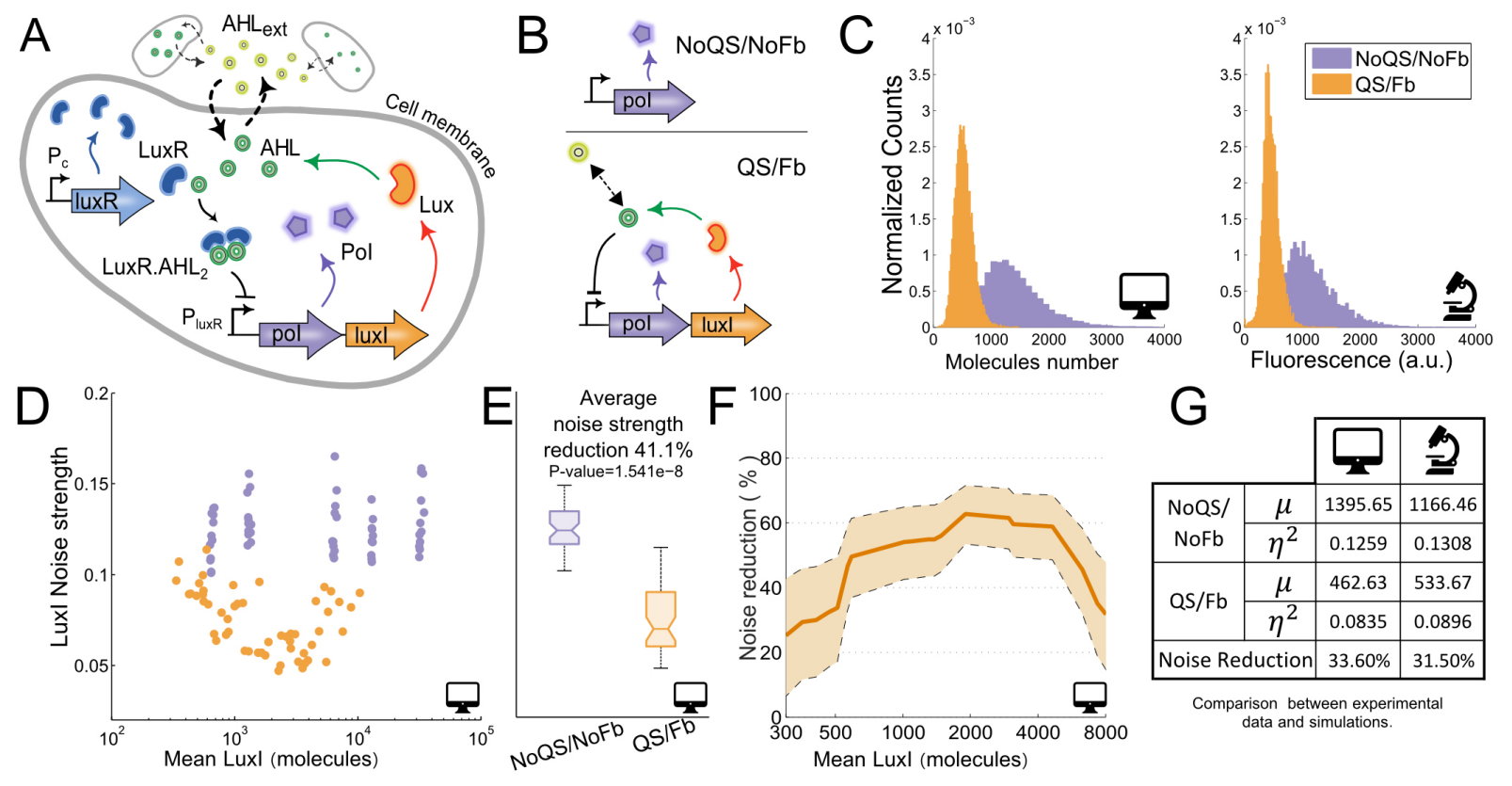

Figura 1: LuxI noise strength under presence/absence of quorum sensing and negative feedback. (A). Proposed synthetic gene circuit. (B) Circuits topologies: NoQS/NoFb (top) and QS/Fb (bottom). (C) Representative computational (left) and experimental (right) population histograms of LuxI noise strength for QS/Fb (orange) presenting a narrower gaussian-like distribution as compared to the Poisson-like one of NoQS/NoFb (purple). From computational simulations: (D) Sampled combinations of LuxI expression parameters for fixed LuxR ones show larger values of LuxI noise strength vs. mean for NoQS/NoFb (purple dots) than for QS/Fb (orange dots). (E) The QS/Fb circuit significantly reduces the average noise strength for the sampled parameters space by $41 \%$, from $\left\langle\eta_{\mathrm{NoQS} / \mathrm{NoFb}}^{2}\right\rangle=0.1263$ down to $\left\langle\eta_{\mathrm{QS} / \mathrm{Fb}}^{2}\right\rangle=0.0744$. (F) For varying LuxI parameters the average reduction of noise strength in LuxI ranges from $30 \%$ up to $60 \%$ and shows dependence on the mean expression level. Data shown for LuxI means between the biotechnological relevant range [300, 8000] molecules/cell. (G) Comparison of experimental and computational statistical moments.

not robust against system uncertainty and/or variations. There is an ever-growing appreciation that biological complexity requires new bioprocess design principles.

Synthetic biology, sometimes defined as the engineering of biology, has the potential to engineer genetic circuits to perform new functions for useful purposes in a systematic, predictable, robust, and efficient way [38]. In the last years, several synthetic circuits have been proposed with the ultimate goal of dealing with gene expression noise $[43,44]$. Though circuits using negative feedback have been proved to decrease gene expression noise [5], single-cell intracellular feedback loops do not take into account that in practice one is interested in controlling gene expression mean value and noise across a population of cells. Feedback across a population of cells can be implemented by means of quorum sensing-based strategies, and has been shown to reduce noise effects [35, 39, 40]. Indeed, cell-to-cell communication by means of quorum sensing induces consensus among cells [27], that is, contributes to reduce the difference of internal state among cells in a population. This, in turn, may contribute to protect from noise [33]. Thus, the idea of joining both intracellular negative feedback and extracellular feedback via quorum sensing is a natural one, that has been suggested in $[1,37,42]$.

In this work we analyze the synthetic gene circuit designed previously in [1] depicted in Fig 1A, designed to reduce gene expression noise while achieving a desired mean expression level in a protein of interest [37]. The circuit uses the repressible promoter $\mathrm{P}_{\text {lux }}$ designed in [7] to implement a negative feedback loop over the gene of interest, and adds a QS mechanism based on N-acyl-Lhomoserine lactone (AHL) $[31,10]$ to induce population consensus (Section 2). We used the stochastic Chemical Langevin Equation [13] to explore the impact of some key circuit parameters on noise strength (Section 3). To assess the role played by feedback and QS we compared the proposed circuit, denoted as $\mathrm{QS} / \mathrm{Fb}$, with constitutive expression (NoQS/NoFb) (Fig. 1B). Extrinsic noise was modeled by randomizing values of the model parameters $[15,36]$. Our in silico analysis (Section 4) reveals significant noise attenuation in gene expression through the interplay between quorum sensing and negative feedback, and explain their 
different roles for different noise sources, highlighting the need for proper characterization of extrinsic noise. Preliminary in vivo (Section 5) results agree with the computational ones.

\section{Circuit description}

The synthetic gene circuit (Fig. 1A) combines two functional subsystems already implemented in $E$. coli. The first subsystem implements a cell-tocell communication mechanism via quorum sensing, based on exchange of the small signaling autoinducer molecule N-acyl-L-homoserine lactone (AHL) [17, 10]. This autoinducer molecule passively diffuses across the cellular membrane to and from the external environment. Intracellular AHL is synthesized by the protein LuxI expressed by an homolog of the gene luxI of $V$. fisheri [31]. The second subsystem uses the synthetic repressible promoter $\mathrm{P}_{\text {lux }}$ designed in [7] to control transcription of the gene luxI. This promoter is repressed by the transcription factor (LuxR.AHL) $)_{2}$. Protein LuxR is expressed by gene luxR under the constitutive promoter $\mathrm{P}_{\mathrm{c}}$. Proteins LuxR and AHL bind creating the heterodimer (LuxR.AHL), which subsequently dimerizes forming the heterotetramer (LuxR.AHL $)_{2}$. This way, the negative feedback control of the LuxI expression is effectively implemented.

\section{Mathematical model}

To analyze how our genetic circuit affects intrinsic and extrinsic noise, we needed an appropriate model and a computationally efficient method. Both aspects are intertwined. We considered an equivalent set of pseudo-reactions resulting from the deterministic model of the circuit, and then derived a stochastic model for a population of $N$ cells whose mean corresponds to that of the deterministic one. We used the Chemical Langevin Equation approach (CLE). Though computationally much more efficient than the Chemical master equation (CME) or even the Gillespie algorithm, the CLE is still computationally demanding when the goal is to simulate a whole population of cells. Since the CLE approximates the CME by a system of stochastic differential equations of order equal to the number of species, a reduced deterministic model with as few species per cell as possible was desirable.

\subsection{Reduced deterministic model}

We aimed at obtaining a reduced model more amenable for computational analysis, but avoiding excessive reduction that would lead to lack of biological relevance. In particular, the species we obtained in the reduced model are not lumped ones. Reduced models accounting for total mRNA and total transcription factor have been proposed to match modeled species with measurable ones [12]. In our case we explicitly modeled bound and unbound forms of the transcription factor, but the model accounts for the total LuxI protein. For our circuit this is a good proxy for the amount of protein of interest if both are co-expressed, and transcriptional noise dominates.

Thus, in a first step we used the mass-action kinetics formalism [4] to get a deterministic model of the full reactions network corresponding to the genetic circuit [1]. We then got a reduced order model by applying the Quasi Steady-State Approximation (QSSA) on the fast chemical reactions and taking into account invariant moieties [1, 20, 24]. The resulting deterministic reduced model is described by equations (1)-(2).

$$
\begin{aligned}
& \dot{n}_{1}^{i}=\frac{\mathrm{C}_{\mathrm{I}} \mathrm{P}_{\mathrm{I}}}{\mathrm{d}_{\mathrm{I}}}\left(\frac{\mathrm{k}_{\mathrm{dlux}}+\alpha n_{3}^{i}}{\mathrm{k}_{\mathrm{dlux}}+n_{3}^{i}}\right)-\mathrm{d}_{\mathrm{I}} n_{1}^{i} \\
& \dot{n}_{2}^{i}=\frac{\mathrm{C}_{\mathrm{R}} \mathrm{P}_{\mathrm{R}}}{\mathrm{d}_{\mathrm{R}}}+\mathrm{k}_{-1} n_{6}^{i}-\left(\frac{\mathrm{k}_{-1}}{\mathrm{k}_{\mathrm{d} 1}} n_{4}^{i}+\mathrm{d}_{\mathrm{R}}\right) n_{2}^{i} \\
& \dot{n}_{3}^{i}=\frac{\mathrm{k}_{-2}}{\mathrm{k}_{\mathrm{d} 2}}\left(n_{6}^{i}\right)^{2}-\left(\mathrm{k}_{-2}+\mathrm{d}_{\mathrm{RA}_{2}}\right) n_{3}^{i} \\
& \dot{n}_{4}^{i}=\mathrm{k}_{-1} n_{6}^{i}+\mathrm{k}_{\mathrm{A}} n_{1}^{i}+\mathrm{D}\left(\mathrm{V}_{\mathrm{c}} n_{5}-n_{4}^{i}\right)-\left(\frac{\mathrm{k}_{-1}}{\mathrm{k}_{\mathrm{d} 1}} n_{2}^{i}+\mathrm{d}_{\mathrm{A}}\right) n_{4}^{i} \\
& \dot{n}_{5}=\mathrm{D}\left(-N \mathrm{~V}_{\mathrm{c}} n_{5}+\sum_{i=1}^{N} n_{4}^{i}\right)-\mathrm{d}_{\mathrm{A}_{\mathrm{e}}} n_{5}
\end{aligned}
$$

with:

$$
\begin{aligned}
& n_{6}^{i}=\frac{\mathrm{k}_{\mathrm{d} 2}\left(\mathrm{~d}_{\mathrm{RA}}+\mathrm{k}_{-1}\right)}{4 \mathrm{k}_{2}}\left[\sqrt{\frac{8 \mathrm{k}_{-2}\left(2 \mathrm{k}_{-2} \mathrm{k}_{\mathrm{d} 1} n_{3}^{i}+\mathrm{k}_{-1} n_{2}^{i} n_{4}^{i}\right)}{\mathrm{k}_{\mathrm{d} 1} \mathrm{k}_{\mathrm{d} 2}\left(\mathrm{~d}_{\mathrm{RA}}+\mathrm{k}_{-1}\right)^{2}}+1}-1\right] \\
& \text { where } \\
& \mathbf{n}(t)^{i}
\end{aligned}
$$

\subsection{Stochastic model}

To model gene expression intrinsic noise we derived a stochastic CLE-based model whose mean corresponds to that of the deterministic reduced model (1)-(2). To this end we first considered the equivalent set of pseudo-reactions (3) for the de- 
terministic model in the $i^{\text {th }}$ cell.

$$
\begin{aligned}
& (\mathrm{LuxR} \cdot \mathrm{AHL})_{2}{\stackrel{f\left(n_{3}, t\right)}{\longrightarrow}}^{\mathrm{LuxI}}+(\mathrm{LuxR} \cdot \mathrm{AHL})_{2} \\
& \text { LuxI } \stackrel{\mathrm{k}_{\mathrm{A}}}{\rightarrow} \mathrm{LuxI}+\mathrm{AHL} \\
& { }^{\mathrm{tt}} \stackrel{\text { Lux R }}{\longrightarrow} \text { LuxR } \\
& \mathrm{LuxR}+\mathrm{AHL} \underset{\mathrm{k}_{-1}}{\stackrel{\mathrm{k}_{-1} / \mathrm{k}_{\mathrm{d}} 1}{\rightleftarrows}} \mathrm{LuxR} \cdot \mathrm{AHL} \\
& 2(\mathrm{LuxR} \cdot \mathrm{AHL}) \underset{g\left(n_{6}, t\right)}{\stackrel{g\left(n_{6}, t\right)}{\rightleftarrows}}(\mathrm{LuxR} \cdot \mathrm{AHL})_{2} \\
& \mathrm{AHL} \underset{\mathrm{DV}_{\mathrm{C}}}{\stackrel{\mathrm{D}}{\rightleftarrows}} \mathrm{AHL}_{\text {ext }} \\
& \operatorname{LuxI} \stackrel{\mathrm{d}_{\mathrm{I}}}{\longrightarrow} \emptyset \\
& \operatorname{LuxR} \stackrel{\mathrm{d}_{\mathrm{R}}}{\longrightarrow} \emptyset \\
& (\mathrm{LuxR} \cdot \mathrm{AHL})_{2} \stackrel{\mathrm{d}_{\mathrm{RA}}}{\longrightarrow} \emptyset \\
& \text { AHL } \stackrel{\mathrm{d}_{\mathrm{A}}}{\rightarrow} \emptyset \\
& \mathrm{AHL}_{\text {ext }} \stackrel{\mathrm{d} A \rho}{\longrightarrow} \emptyset
\end{aligned}
$$

where we denoted $f\left(n_{3}^{i}, t\right) \triangleq \frac{\mathrm{C}_{\mathrm{I}} \mathrm{p}_{\mathrm{I}}}{\mathrm{dm}_{\mathrm{I}}}\left(\frac{\mathrm{k}_{\mathrm{dlux}}+\alpha_{\mathrm{I}} n_{3}^{i}}{\mathrm{k}_{\mathrm{dlux}}+n_{3}^{i}}\right)$ as the Hill-like function associated to LuxI expression, $g\left(n_{6}^{i}, t\right)$ corresponds to the dimerization reflected in equation $(2)$, and $\mathrm{tt}_{\mathrm{LuxR}}=\frac{\mathrm{C}_{\mathrm{R}} \mathrm{P}_{\mathrm{R}}}{\mathrm{dm}_{\mathrm{R}}}$ represent the transcription-translation activity of luxI and lux $R$ respectively, $\mathrm{V}_{\mathrm{c}}=\frac{\mathrm{V}_{\text {cell }}}{\mathrm{V}_{\text {ext }}}$ is the ratio between the cell volume and the culture medium volume, and $\emptyset$ denotes species degradation.

For the computational analysis we used the EulerMaruyama discretization (4) of the stochastic model resulting from the set of pseudo-reactions (3) :

$$
\mathbf{n}(t+\delta t)=\mathbf{n}(t)+\mathbf{S} \cdot \mathbf{a}(\mathbf{n}) \delta t+\mathbf{S} \cdot \mathcal{N} \cdot \sqrt{\mathbf{a}(\mathbf{n})} \sqrt{\delta t},
$$

where $\mathbf{n}(t)=\left[\mathbf{n}(t)^{i}, \ldots \mathbf{n}(t)^{\mathrm{N}}, n_{5}\right]^{T}$ are the number of molecules of each species in the population. The stoichiometry matrix $\mathbf{S}$, whose elements are the stoichiometry submatrices for each cell $\mathbf{S}_{\text {cell }}$ and the external stoichiometry $\mathbf{S}_{\mathbf{e x t}}$, has structure:

$$
\mathbf{S}=\left[\begin{array}{c|c}
\mathbf{S}_{\mathbf{c e l l}} \otimes \mathbf{I}_{\mathbf{N}} & \mathbf{0}_{\mathbf{N} \times \mathbf{1}} \\
\hline \mathbf{S}_{\mathbf{e x t}} \otimes \mathbf{1}_{\mathbf{1} \times \mathbf{N}} & -1
\end{array}\right],
$$

where $\otimes$ is the Kronecker product, $\mathbf{I}_{\mathbf{N}}$ the identity matrix of dimension $N \times N, \mathbf{0}_{\mathbf{N} \times \mathbf{1}}$ and $\mathbf{1}_{\mathbf{1} \times \mathbf{N}}$ are vectors of zeroes and ones respectively, and the coefficients in the stoichiometry matrices $\mathbf{S}_{\text {cell }}$ and $\mathbf{S}_{\text {ext }}$, obtained from the set of pseudo-reactions (3), are:

$$
\begin{aligned}
\mathbf{S}_{\text {cell }} & =\left[\begin{array}{ccccccccccccc}
1 & -1 & 0 & 0 & 0 & 0 & 0 & 0 & 0 & 0 & 0 & 0 & 0 \\
0 & 0 & 1 & 1 & -1 & -1 & 0 & 0 & 0 & 0 & 0 & 0 & 0 \\
0 & 0 & 0 & 0 & 0 & 0 & 1 & -1 & -1 & 0 & 0 & 0 & 0 \\
0 & 0 & 0 & 1 & -1 & 0 & 0 & 0 & 0 & 1 & -1 & -1 & 1
\end{array}\right] \\
\mathbf{S}_{\text {ext }} & =\left[\begin{array}{llllllllllllll}
0 & 0 & 0 & 0 & 0 & 0 & 0 & 0 & 0 & 0 & 0 & 1 & -1
\end{array}\right] .
\end{aligned}
$$

The term $\mathbf{a}(\mathbf{n})$ in (4) is the associated vector of reaction propensities for the whole population of cells, with:

$$
\begin{aligned}
\mathbf{a}(\mathbf{n})=\left[\begin{array}{llllll}
\mathbf{a}(\mathbf{n})^{1} & \mathbf{a}(\mathbf{n})^{2} & \ldots & \mathbf{a}(\mathbf{n})^{N} & \mid & \mathrm{d}_{\mathrm{A}_{\mathrm{e}}} n_{5}
\end{array}\right]^{T} \\
\mathbf{a}(\mathbf{n})^{i}=\left[\begin{array}{llllll}
f\left(n_{3}^{i}, t\right) & \mathrm{d}_{\mathrm{I}} n_{1}^{i} & \frac{\mathrm{C}_{\mathrm{R}} \mathrm{PR}_{\mathrm{R}}}{\mathrm{dm}_{\mathrm{R}}} & \mathrm{k}_{-1} n_{6}^{i} & \frac{\mathrm{k}_{-1}}{\mathrm{k}_{\mathrm{d} 1} n_{2}^{i} n_{4}^{i}} \\
\mathrm{~d}_{\mathrm{R}} n_{2}^{i} & \frac{\mathrm{k}_{-2}}{\mathrm{k}_{\mathrm{d} 2}}\left(n_{6}^{i}\right)^{2} & \mathrm{k}_{-2} n_{3}^{i} & \mathrm{~d}_{\mathrm{RA}_{2} n_{3}} n^{i} & \mathrm{k}_{\mathrm{A}} n_{1}^{i} \\
\mathrm{~d}_{\mathrm{A}} n_{4}^{i} & \mathrm{D} n_{4}^{i} & \mathrm{DV}_{\mathrm{c}} n_{5}
\end{array}\right]^{T}
\end{aligned}
$$

Finally, $\mathcal{N}_{(\mathbf{J N}+\mathbf{1}) \times(\mathbf{J N}+\mathbf{1})}$, where $J=13$ is the number of reactions for the $i^{\text {th }}$ cell, is a diagonal matrix of continuous normal random variables with zero mean and unit variance.

Notice we used lumped propensity functions derived from the reduced model, like the $f\left(n_{3}\right)$ Hilllike function associated to LuxI repression. This approach has already been used in [41]. We validated it for our model by simulating the pseudoreaction associated to $f\left(n_{3}^{i}\right)$ using CLE, and comparing the result with that obtained by simulating the set of corresponding original reactions using Gillespie's direct method SSA [1].

Extrinsic noise was modeled by randomizing the values of the model parameters $[15,36]$, an approach that can easily be integrated within the CLE framework. We assumed a normal distribution to generate the model parameters of the $i^{\text {th }}$ cell in the population.

The stochastic simulations we performed for 400 $\min$ using $\delta t=25 \cdot 10^{-4} \mathrm{sec}$.

\section{Computational analysis}

We used the stochastic model (4) of the proposed circuit, hereafter denoted as circuit $\mathrm{QS} / \mathrm{Fb}$, to explore the impact of some key circuit parameters on noise. As control circuit to compare with, we considered a second circuit which removes both QS and the feedback loop, denoted as NoQS/NoFb. For the computational analysis, this accounts to setting the synthesis of AHL to zero $\left(\mathrm{k}_{\mathrm{A}}=0 \mathrm{~min}^{-1}\right)$ in model (4). This condition is achieved in the lab experimental implementation by taking out the gene coding for LuxI (Section $5)$.

Gene expression noise was evaluated using the squared coefficient of variation, i.e. the noise strength measure $\left(\eta^{2}=(\sigma / \mu)^{2}\right)$. This measure properly captures the contributions of both intrinsic and extrinsic noise [23], and allows comparisons for different expression rates.

We followed the following general procedure. First, for different combinations of the model parameters, we performed temporal simulations of the number of molecules of each species in the circuit for every cell in the population of our sys- 
tem. Extrinsic noise was modeled by randomizing the values of the model parameters using a normal distribution with a variance of $15 \%$. The models were implemented using OpenFPM (http://openfpm.mpi-cbg.de), a C++ version of the Parallel Particle Mesh (PPM) library allowing efficient computational particle-mesh simulations [30]. The code is available in (http: //sb2cl.ai2.upv.es/content/software). In all simulations we used a population of $N=240$ cells in a culture volume of $10^{-3} \mu \mathrm{l}$, corresponding to an optical cell density $\mathrm{OD}_{600}=0.3$.

Then, we obtained the first two statistical moments $\mu$ and $\sigma^{2}$ for each species in the cell population at every time $t_{\mathrm{k}}$. We used the laws of total expectation and total variance. From these moments, we calculated long-term distributions to infer the noise strength of each species.

Finally, we explored the effect of variations in parameters associated to expression of LuxI and LuxR, as they are as key parameters in our circuit. For LuxI, we considered the dissociation constant $\mathrm{k}_{\text {dlux }}$ between the transcription factor $(\mathrm{LuxR} \cdot \mathrm{AHL})_{2}$ and the repressible $\mathrm{P}_{\text {lux }}$ promoter, the translation rate $\mathrm{p}_{\mathrm{I}}$, and the basal expression $\alpha_{\mathrm{I}}$ of the $\mathrm{P}_{\text {lux }}$ promoter. We sampled in the ranges $\mathrm{k}_{\text {dlux }}=[10-2000]$ molecules, $\alpha=[0.01-0.1]$, and $\mathrm{p}_{\mathrm{I}}=[0.2-10] \mathrm{min}^{-1}$ selected from the literature $[28,6,32]$ and experimentally achievable in the lab. As for LuxR, we considered two values for the the translation rate $\mathrm{p}_{\mathrm{R}}$ : a strong $\mathrm{RBS}\left(\mathrm{p}_{\mathrm{R}}=10\right.$ $\left.\min ^{-1}\right)$, and a medium-weak one $\left(\mathrm{p}_{\mathrm{R}}=2 \mathrm{~min}^{-1}\right)$.

\section{Strains, plasmids and experimental protocol}

To validate the in silico computational results, we implemented the QS/Fb and NoQS/NoFb circuits in vivo. We used components from the iGEM Registry of Standard Biological Parts. All parts were cloned using the Biobrick's foundation 3 Antibiotic Assembly method. All coding sequences have the double-terminator BBa_B0015, and were confirmed by sequencing. The circuit QS/Fb couples both QS-based cell-to-cell communication and the negative feedback subsystems. It was split in two subunits integrated in different plasmids.

On the one hand, plasmid pCB2tc contains the gene luxR (part BBa_C0062) coding for the protein LuxR constitutively expressed under the control of a medium strength promoter (part BBa_J23106), and a strong RBS (part BBa_B0034).

This insert was cloned into the pACYC184 plasmid cloning vector (p15A origin, 10-12 copies/cell, chloramphenicol/tetracycline [29]).
On the other hand, plasmid pYB06ta contains gene luxI (part BBa_C0161) under control of the $\mathrm{P}_{\text {luxR }}$ repressible promoter (part BBa_R0062) and a strong RBS (part BBa_B0034). The strong RBS BBa_B0034 and the green fluorescent protein (GFP, part BBa_E0040) were inserted using GIBSON assembly (NEB Catalog Number E2611S) upstream of luxI, right after the $\mathrm{P}_{\text {luxR }}$ promoter. This way, GFP, used as protein of interest ( $\mathrm{PoI}$ in Fig. 1A) is co-expressed with LuxI.

They were inserted into the pBR322 plasmid cloning vector (pMB1 origin, 15-20 copies/cell, ampicillin/tetracycline [29]). Finally, both plasmids pCB2tc and pYB06ta were co-transformed in competent cells (DH- $5 \alpha$, Invitrogen). Notice being both plasmids low copy ones, they do not introduce a big metabolic burden on the cell. On the other hand, their variability is quite narrow so gene copy number will not be the only relevant extrinsic noise source in the experimental setup.

As control network, we implemented the circuit NoQS/NoFb which removes both QS and the feedback loop. To this end, the plasmid pCB2tc above was co-transformed with the plasmid pAV02ta (pMB1 origin, ampicillin/tetracycline) containing only GFP downstream of the $\mathrm{P}_{\text {luxR }}$ repressible promoter (part BBa_R0062) and the the strong RBS (part BBa_B0034). Both were cloned in the pBR322 plasmid cloning vector.

\subsection{Experimental protocol}

For the experimental validation of the circuit (protocol details are in [1]), two sets of E. coli cells (cloning strain DH-5 $\alpha$ ) carrying the $\mathrm{QS} / \mathrm{Fb}$ and $\mathrm{NoQS} / \mathrm{NoFb}$ circuits respectively, were inoculated from $-80^{\circ} \mathrm{C}$ stocks into $3 \mathrm{~mL}$ of $\mathrm{LB}$ with appropriate antibiotics, followed by an overnight incubation at $37{ }^{\circ} \mathrm{C}$ and $250 \mathrm{rpm}$ in $14 \mathrm{ml}$ culture tubes. When the cultures reached an optical density (OD) of 4 (600 nm, Eppendorf BioPhotometer D30), the overnight cultures were diluted 500-fold $\left(\mathrm{OD}_{600}\right.$ of 0.02$)$ into M9 medium with appropriate antibiotics. These were used to inoculate new cultures, which were incubated for 7 hours $\left(37^{\circ} \mathrm{C}\right.$, $250 \mathrm{rpm}, 14 \mathrm{ml}$ culture tubes) until they reached an $\mathrm{OD}_{600}$ between $0.2-0.3$. At this point, cell growth and protein expression were interrupted by transferring the culture into an ice-water bath for 10 min. Next, $50 \mu \mathrm{L}$ of each tube were transferred into $1 \mathrm{ml}$ of phosphate-buffered saline with 500 $\mu \mathrm{g} / \mathrm{mL}$ of the transcription inhibitor rifampicin (PBS + Rif) in one $5 \mathrm{~mL}$ cytometer tube, and incubated during 1 hour in a water bath at $37^{\circ} \mathrm{C}$, so that transcription kept blocked and GFP had time to mature and fold properly. Samples were measured at different time points using the BD 
FACSCalibur flow cytometer (original default configuration parameters), and flow cytometry data analyzed with custom scripts.

\section{Results}

We first addressed the question whether the proposed $\mathrm{QS} / \mathrm{Fb}$ circuit effectively reduces noise strength with respect to the circuit NoQS/NoFb (Fig. 1B). The last one consists of the LuxR expression on the one hand, and the protein of interest (PoI) downstream the $\mathrm{P}_{\text {lux }}$ repressible promoter, without the luxI gene coding for LuxI protein, on the other. Since no autoinducer $A H L$ is neither produced nor externally introduced, there is no repression, so the expression of PoI is essentially a constitutive one (Section 2). This corresponds to the Poisson distribution observed in the purple population histogram in the left panel of Fig. 1C. Contrarily, the QS/Fb histogram departs from the Poisson distribution to become a narrow Gaussian-like one in the orange population histogram in the left panel of Fig. 1C. This fact, and the reduction in the mean expression value, indicate the strong presence of regulation. In both cases we used the nominal circuit parameters [1].

Reduction in noise strength was not due to a particular choice of the circuit parameter values, but a property of the proposed topology. Fig. 1D depicts LuxI noise strength vs. mean expression for 60 different combinations of the $\mathrm{P}_{\mathrm{LuxR}}$ characteristics for both QS/Fb (orange points) and NoQS/NoFb (purple points). The points in the figure correspond to the mean values across the cells population for each combination of parameters (Section 4). The magnitude of noise strength reduction was larger for medium values of mean protein expression. Noise strength levels were similar for all mean expression values in the case of the NoQS/NoFb circuit. Mean expression values in this case depend only on the translation rate $\mathrm{p}_{\mathrm{I}}$ for which five discrete values were used, inducing the five mean values seen in the figure. On the contrary, the QS/Fb circuit showed lower values of noise strength and more graded values of the mean expression level, as it depends on the combination of all three parameters varied.

More important, noise strength was consistently lower for the QS/Fb circuit. Taking together all the different combinations of promoter parameters for each circuit, and the average noise strength was significantly reduced by $41 \%$ in the presence of quorum sensing and negative feedback as shown in Fig. 1E.

For the given fixed LuxR expression parameters, the noise strength reduction in LuxI showed a clear dependence on its mean expression level. In Fig. $1 \mathrm{~F}$ the minimum and maximum values of LuxI noise reduction are plotted as a function of its mean value. In the range between 600 and 6000 LuxI molecules it was possible to reduce the noise variance at least in $35 \%$ in the worst case scenario, with a maximum reduction of around $70 \%$ for means between 2000 and 3000 molecules.

\section{Conclusion}

Our results show that gene synthetic circuits benefiting from the interplay between feedback and cell-to-cell communication allow control of the mean expression level and noise strength of a protein of interest. A few circuit parameters easy to tune in the wet-lab can be used to achieve noise strength reductions up to a $60 \%$ with respect to constitutive expression of the protein of interest.

Mean expression level and noise strength are not independent goals. At low mean values intrinsic noise dominates and sets the minimum noise strength attainable. At high mean values extrinsic noise dominates. Thus, there is a trade-off between expression level and noise strength, as revealed both by system-wide experimental data and theoretical analysis reported in the literature. Our computational results fitted well in this scenario, and suggest that tuning synthetic gene circuits to minimize noise while achieving a desired expression level will require a multi-objective optimization approach.

The experimental results, though preliminary, showed a high concordance the computational ones and confirmed the capability of the proposed circuit to reduce noise strength.

\section{Agradecimientos}

This work was partially supported by the Spanish Government (CICYT DPI2014-55276-C5-1) and the European Union (FEDER). Y.B. thanks grant FPI/2013-3242 of UPV.

\section{Referencias}

[1] Yadira Boada, Alejandro Vignoni, and Jesus Pico. Engineered control of genetic variability reveals interplay between quorum sensing, feedback regulation and biochemical noise. ACS Synthetic Biology, 0(Just Accepted):null, 0 .

[2] M. Carlquist, R. L. Fernandes, S. Helmark, Anna-Lena L. Heins, L. Lundin, S. J. Sorensen, K.V. Gernaey, and A. E. Lantz. Physiological heterogeneities in microbial populations and implications for physical 
stress tolerance. Microb Cell Fact, 11:94, 2012 .

[3] Guilhem Chalancon, Charles N. J. Ravarani, S. Balaji, Alfonso Martinez-Arias, L. Aravind, Raja Jothi, and M. Madan Babu. Interplay between gene expression noise and regulatory network architecture. Trends Genet, 28(5):221-32, 52012.

[4] Vijaysekhar Chellaboina, Sanjay Bhat, Wassim Haddad, and Dennis Bernstein. Modeling and analysis of mass-action kinetics. IEEE Control Systems Magazine, 29(4):6078, 82009 .

[5] Yann Dublanche, Konstantinos Michalodimitrakis, Nico Kümmerer, Mathilde Foglierini, and Luis Serrano. Noise in transcription negative feedback loops: simulation and experimental analysis. Mol Syst Biol, 2:41, 2006.

[6] Robert G Egbert and Eric Klavins. Finetuning gene networks using simple sequence repeats. Proceedings of the National Academy of Sciences of the United States of America, 2012.

[7] K. A. Egland and E. P. Greenberg. Conversion of the vibrio fischeri transcriptional activator, luxr, to a repressor. Journal of Bacteriology, 182(3):805-811, 2000.

[8] Avigdor Eldar and Michael B. Elowitz. Functional roles for noise in genetic circuits. $\mathrm{Na}$ ture, 467(7312):167-173, 2010.

[9] R. Lencastre Fernandes, M. Nierychlo, L. Lundin, A. E. Pedersen, P. E. Puentes Tellez, A. Dutta, M. Carlquist, A. Bolic, D. Schäpper, A. C. Brunetti, S. Helmark, A-L L. Heins, A. D. Jensen, I. Nopens, K. Rottwitt, N. Szita, J. D. van Elsas, P. H. Nielsen, J. Martinussen, S. J. Sorensen, A. E. Lantz, and K. V. Gernaey. Experimental methods and modeling techniques for description of cell population heterogeneity. Biotechnol Adv, 29(6):575-99, 2011.

[10] C. Fuqua, M.R. Parsek, and E.P. Greenberg. Regulation of gene expression by cellto-cell communication: acyl-homoserine lactone quorum sensing. Annual review of genetics, 35(1):439-468, 2001.

[11] K. A. Geiler-Samerotte, C. R. Bauer, S. Li, N. Ziv, D. Gresham, and M. L. Siegal. The details in the distributions: why and how to study phenotypic variability. Curr Opin Biotechnol, 24(4):752-9, 82013.
[12] Edward J. Hancock, Guy-Bart B. Stan, James A. J. Arpino, and Antonis Papachristodoulou. Simplified mechanistic models of gene regulation for analysis and design. J R Soc Interface, 12(108):20150312, 7 2015.

[13] Desmond J. Higham. Modeling and simulating chemical reactions. SIAM Review, 50(2):347-368, 2008.

[14] Daniel L. Jones, Robert C. Brewster, and Rob Phillips. Promoter architecture dictates cell-to-cell variability in gene expression. Science, 346(6216):1533-6, 122014.

[15] Jaewook Joo, Steven J. Plimpton, and JeanLoup L. Faulon. Statistical ensemble analysis for simulating extrinsic noise-driven response in nf- $v \mathrm{~b}$ signaling networks. BMC Syst Biol, $7: 45,2013$

[16] Mads Kærn, Timothy C. Elston, William J. Blake, and James J. Collins. Stochasticity in gene expression: from theories to phenotypes. Nat Rev Genet, 6(6):451-64, 2005.

[17] H. B. Kaplan and E. P. Greenberg. Diffusion of autoinducer is involved in regulation of the vibrio fischeri luminescence system. Journal of bacteriology, 163(3):1210-1214, 1985.

[18] Kyung H. Kim and Herbert M. Sauro. Adjusting phenotypes by noise control. PLoS Comput Biol, 8(1):e1002344, 2012.

[19] Piyush Labhsetwar, John Andrew Cole, Elijah Roberts, Nathan D. Price, and Zaida A. Luthey-Schulten. Heterogeneity in protein expression induces metabolic variability in a modeled escherichia coli population. Proceedings of the National Academy of Sciences, 110(34):14006-14011, 2013.

[20] Bence Mélykúti, João P. Hespanha, and Mustafa Khammash. Equilibrium distributions of simple biochemical reaction systems for time-scale separation in stochastic reaction networks. $J R$ Soc Interface, 11(97):20140054, 82014.

[21] Susann Müller, Hauke Harms, and Thomas Bley. Origin and analysis of microbial population heterogeneity in bioprocesses. Curr Opin Biotechnol, 21(1):100-13, 2010.

[22] Edward M. Nelson, Volker Kurz, Nicolas Perry, Douglas Kyrouac, and Gregory Timp. Biological noise abatement: Coordinating the responses of autonomous bacteria in a synthetic biofilm to a fluctuating environment using a stochastic bistable switch. ACS synthetic biology, 3(5):286-297, 2013. 
[23] Johan Paulsson. Summing up the noise in gene networks. Nature, 427(6973):415-8, 1 2004.

[24] Jesús Picó, Alejandro Vignoni, Enric PicóMarco, and Yadira Boada. Modelling biochemical systems: from mass action kinetics to linear noise approximation. Revista Iberoamericana de Automática e Informática Industrial RIAI, 12(3):241-252, 72015.

[25] Arjun Raj and Alexander van Oudenaarden. Nature, nurture, or chance: stochastic gene expression and its consequences. Cell, 135(2):216-226, 2008.

[26] Jonathan M Raser and Erin K O'Shea. Noise in gene expression: origins, consequences, and control. Science, 309(5743):2010-2013, 2005.

[27] Giovanni Russo and Jean Jacques E. Slotine. Global convergence of quorum-sensing networks. Phys Rev E Stat Nonlin Soft Matter Phys, 82(4 Pt 1):041919, 102010.

[28] Howard M Salis, Ethan A Mirsky, and Christopher A Voigt. Automated design of synthetic ribosome binding sites to control protein expression. Nature biotechnology, 27(10):946-950, 2009.

[29] Sambrook et al. Molecular cloning: a laboratory manual. Number Ed. 2. Cold spring harbor laboratory press, 1989.

[30] I. F. Sbalzarini, J. H. Walther, M. Bergdorf, S. E. Hieber, E. M. Kotsalis, and P. Koumoutsakos. Ppm- a highly efficient parallel particle-mesh library for the simulation of continuum systems. Journal of Computational Physics, 215(2):566-588, 2006.

[31] Amy L. Schaefer, Dale L. Val, Brian L. Hanzelka, J. E. Cronan, and E. P. Greenberg. Generation of cell-to-cell signals in quorum sensing: acyl homoserine lactone synthase activity of a purified vibrio fischeri luxi protein. Proceedings of the National Academy of Sciences, 93(18):9505-9509, 1996.

[32] Sebastian R. Schmidl, Ravi U. Sheth, Andrew $\mathrm{Wu}$, and Jeffrey J. Tabor. Refactoring and optimization of light-switchable escherichia coli two-component systems. ACS synthetic biology, 3(11):820-831, 2014.

[33] Nicolas Tabareau, Jean-Jacques J. Slotine, and Quang-Cuong C. Pham. How synchronization protects from noise. PLoS Comput Biol, 6(1):e1000637, 2010.
[34] Omar P. Tabbaa, German Nudelman, Stuart C. Sealfon, Fernand Hayot, and Ciriyam Jayaprakash. Noise propagation through extracellular signaling leads to fluctuations in gene expression. BMC Syst Biol, 7:94, 2013.

[35] Yu Tanouchi, Dennis Tu, Jungsang Kim, and Lingchong You. Noise reduction by diffusional dissipation in a minimal quorum sensing motif. PLoS Comput Biol, 4(8):e1000167, 2008.

[36] Tina Toni and Bruce Tidor. Combined model of intrinsic and extrinsic variability for computational network design with application to synthetic biology. PLoS Comput Biol, 9(3):e1002960, 2013.

[37] A. Vignoni, D. A. Oyarzún, J. Picó, and G. B. Stan. Control of protein concentrations in heterogeneous cell populations. Procs. 2013 European Control Conference (ECC), pages 3633-3639, 2013.

[38] Jeffrey C. Way, James J. Collins, Jay D. Keasling, and Pamela A. Silver. Integrating biological redesign: where synthetic biology came from and where it needs to go. Cell, 157(1):151-61, 2014.

[39] Marc Weber and Javier Buceta. Noise regulation by quorum sensing in low mrna copy number systems. BMC Syst Biol, 5:11, 2011.

[40] Marc Weber and Javier Buceta. Dynamics of the quorum sensing switch: stochastic and non-stationary effects. BMC Syst Biol, 7:6, 2013.

[41] Mae L. Woods, Miriam Leon, Ruben PerezCarrasco, and Chris P. Barnes. A statistical approach reveals designs for the most robust stochastic gene oscillators. ACS Synth Biol, 22016 .

[42] Amin Zargar, David N. Quan, and William E. Bentley. Enhancing intercellular coordination: Rewiring quorum sensing networks for increased protein expression through autonomous induction. ACS Synth Biol, $5(9): 923-8,2016$.

[43] Christoph Zechner, Georg Seelig, Marc Rullan, and Mustafa Khammash. Molecular circuits for dynamic noise filtering. Proceedings of the National Academy of Sciences, page 201517109, 42016.

[44] Carolyn Zhang, Ryan Tsoi, and Lingchong You. Addressing biological uncertainties in engineering gene circuits. Integrative Biology, 8(4):456-464, 2016. 